\title{
Ảnh hưởng của lãnh đạo tư lợi đến sự kiệt sức về tinh thần, với vai trò trung gian của sự hoài nghi đối với tổ chức: Trường hợp người lao động trong doanh nghiệp Thành phố Hồ Chí Minh
}

\section{The effect of Machiavellian leadership on employees' emotional exhaustion, organizational cynicism as a mediator: Evidence from Ho Chi Minh city}

Trần Minh Duy ${ }^{1 *}$

${ }^{1}$ Trường Đại học Sài Gòn, Việt Nam

*Tác giả liên hệ, Email: tmduy@ sgu.edu.vn

THÔNG TIN

TÓM TÁT

DOI: $10.46223 /$ HCMCOUJS. econ..

Ngày nhận: 15/03/2021

Ngày nhận lại: 09/05/2021

Duyệt đăng: 28/05/2021

Tù khóa:

lãnh đạo tư lợi; sự hoài nghi đối với tổ chức; sự kiệt sức về tinh thần

Keywords:

machiavellian leadership; organizational cynicism; emotional exhaustion
Lãnh đạo tư lợi, sự hoài nghi đối với tổ chức và sự kiệt sức về tinh thần đang là những chủ đề được quan tâm gần đây, vì chúng gây ra ảnh hưởng tiêu cực đến các mặt quan trọng của tổ chức. Nghiên cứu này nhằm kiểm định lại các mối quan hệ trên tại bối cảnh ở Việt Nam và củng cố những kết quả của các nghiên cứu trước đây trên thế giới. Các mối quan hệ này được kiểm định thông qua mẫu khảo sát 336 người lao động đang làm việc tại Thành phố Hồ Chí Minh. Kết quả nghiên cứu cho thấy lãnh đạo tư lợi có tác động cùng chiều trực tiếp và gián tiếp đến sự kiệt sức về tinh thần thông qua vai trò trung gian của sự hoài nghi đối với tổ chức, và kết quả này phù hợp kết quả của các nghiên cứu trước đây. Từ đó, nghiên cứu này đã đóng góp những bằng chứng thực nghiệm về mối quan hệ giữa các khái niệm này. Ngoài ra, nghiên cứu cũng đưa ra một số hàm ý quản trị và giải pháp liên quan cho tổ chức về tăng cường hiệu quả lãnh đạo và giảm thiểu các ảnh hưởng tiêu cực.

ABSTRACT
Machiavellian leadership, organizational cynicism and
emotional exhaustion are increasingly attracting interest due to their
negative effects on organizational key outcomes. The aim of this
study is to examine the relationships of these constructs in Vietnam
context and strengthen the previous results. Specifically, this study
used a sample of 336 employees working in Ho Chi Minh city. The
result which is consistent with previous findings shows that
Machiavellian leadership affects emotional exhaustion both directly
and indirectly through organizational cynicism. Thus, this study
suggests that there is good evidence to support relationships among
the three factors above. This research also provides some managerial
related implications to improve leadership's effectiveness and reduce
organizational cynicism and emotional exhaustion.




\section{Giới thiệu}

Trong những thời gian gần đây, stress công việc đã là một khái niệm dần quen thuộc với nhiều người, và tác hại của nó là điều không thể chối cãi. Khi phải chịu đựng stress công việc trong thời gian dài sẽ dẫn đến một hội chứng, đó là cạn kiệt sức lực vì công việc (burnout). Hội chứng này có nhiều nguyên nhân nhưng thể hiện nhưng nổi bật và quan trọng nhất vẫn là sự kiệt sức về tinh thần (Cropanzano, Rupp, \& Byrne, 2003; Maslach, Schaufeli, \& Leiter, 2001). Khi đề cập đến các nguyên nhân liên quan đến stress công việc và sự kiệt sức về tinh thần, các nghiên cứu gần đây cũng cho thấy rằng có rất nhiều yếu tố, trong đó có cả lãnh đạo và lòng tin đối với tổ chức của người lao động (Chiaburu, Peng, Oh, Banks, \& Lomeli, 2013; LeBreton, Shiverdecker, \& Grimaldi, 2018; Lee \& Ashforth, 1996). Mặt khác, một số nhà nghiên cứu nhận định rằng so với các tác động tích cực thì các tác động tiêu cực đến tổ chức là mạnh hơn (Chiaburu et al., 2013). Điều này có thể lý giải là vì bản chất tâm lý con người có xu hướng phản mạnh với những tác động tiêu cực hơn là tích cực (Baumeister, Bratslavsky, Finkenauer, \& Vohs, 2001; Grichnik, Smeja, \& Welpe, 2010; Hobfoll, 1989). Vì thế vấn đề được đặt ra là khi tìm hiểu về các nguyên nhân gây ra sự kiệt sức về tinh thần thì các yếu tố tiêu cực của tổ chức cũng cần phải được xem xét?

Thực tế trên thế giới những yếu tố tiêu cực của tổ chức nói trên đã được đề cập trong nghiên cứu của những thập niên trước đây. Xét về lãnh đạo, nhân cách tối của lãnh đạo nhận được sự quan tâm lớn, đặc biệt là lãnh đạo tư lợi, điển hình như nghiên cứu của Christie và Geis (1970) về nhóm nhân cách này. Xét về mặt lòng tin với tổ chức, sự hoài nghi đối với tổ chức đang là một vấn đề nổi cộm (Chiaburu et al., 2013) vì nó đang có xu hướng gia tăng và xuất hiện khắp nơi trong tổ chức (Thomas \& Gupta, 2018). Nghiên cứu của Dean, Brandes, và Dharwadkar (1998) đã mô tả chi tiết và đưa ra định nghĩa về sự hoài nghi này. Các vấn đề về nguyên nhân và hệ quả của sự kiệt sức về tinh thần hay hội chứng cạn kiệt sức lực cũng được dần được các nhà nghiên cứu khai phá (Hobfoll, 1989; Lee \& Ashforth, 1996; Maslach \& Jackson, 1981).

Thêm vào đó, các tác động tiêu cực lên tổ chức của các yếu tố này cũng đã được các nhà nghiên cứu tập trung tìm hiểu. Về lãnh đạo với ba mặt của nhân cách tối, Spain, Harms, và LeBreton (2014) đề xuất mô hình nhằm giải thích về cơ chế tác động của lãnh đạo với ba mặt tối, tuy nhiên các tác giả này chỉ mới đề xuất mô hình ban đầu và cho rằng cần có nhiều nghiên cứu ở các bối cảnh khác nhau để khẳng định mạnh mẽ hơn. Nghiên cứu của Volmer, Koch, và Göritz (2016) cho thấy mỗi một mặt trong nhân cách tối của lãnh đạo đều có ảnh hưởng đến nhân viên cấp dưới của họ, cụ thể là sự thành công trong nghề nghiệp và hành xử của nhân viên cấp dưới. Nghiên cứu của LeBreton và cộng sự (2018) trên cơ sở tổng hợp kết quả của các nghiên cứu trước đây đã chỉ ra rằng các nhân tối của lãnh đạo đều có ảnh hưởng đến các mặt của tổ chức như công tác của chính lãnh đạo, kết quả đầu ra của tổ chức, thái độ trong công việc của nhân viên và cả tác động qua lại với nhau của lãnh đạo - nhân viên đồng thời mang nhân cách tối. Về sự hoài nghi đối với tổ chức, nghiên cứu tổng hợp của Chiaburu và cộng sự (2013) thể hiện được các tiền tố và hệ quả của sự hoài nghi này. Các tiền tố đáng chú ý có thể kể đến như: các trải nghiệm làm việc, khuynh hướng của nhân viên, các yếu tố thuộc về nhân khẩu học; và các hệ quả tiêu cực đó chính là thái độ và hành vi của nhân viên. Về sự kiệt sức về tinh thần, nghiên cứu của Lee và Ashforth (1996) đã tập trung làm rõ và giải thích cơ chế tác động của từng thành phần trong hội chứng cạn kiệt sức lực. Kết quả nghiên cứu cũng cho thấy hội chứng cạn kiệt sức lực cũng như sự kiệt sức về tinh thần có các tiền tố tương tự nhau. Mặc dù các nghiên cứu này đã được thực hiện tại nhiều bối cảnh khác nhau, tuy nhiên cũng như để có cơ sở khẳng định mạnh mẽ hơn thì cần có nhiều nghiên cứu hơn nữa về các vấn đề này. Và thực tế rằng các nghiên cứu về các khái niệm kể trên vẫn chưa xuất hiện nhiều tại các bối cảnh của các nước Châu Á, đặc biệt là Việt Nam.

Tại Việt Nam, nghiên cứu liên quan đến các khái niệm liên quan đến các vấn đề này vẫn 
còn khá ít và có vẻ như chưa được quan tâm nhiều. Khi đề cập đến hội chứng cạn kiệt sức lực có thể kể đến nghiên cứu của Nguyen, Kitaoka, Sukigara, và Thai (2018). Nghiên cứu này được thực hiện tại ba bệnh viện lớn ở Việt Nam, với mẫu khảo sát là có số lượng 430 y tá, với mục đích nhằm kiểm định lại yếu tố điều khiển trong công việc ảnh hưởng thế nào đến ba thành phần của hội chứng cạn kiệt sức lực, tuy nhưng chưa xem xét đến các tác động của chúng các đến mặt của tổ chức. Xét về lãnh đạo, các nghiên cứu tại Việt Nam nhìn chung vẫn theo định hướng về các mặt tích cực của lãnh đạo như về phẩm chất và phong cách lãnh đạo. Điển hình như nghiên cứu của Mai và Nguyen (2015) với mục đích nhằm tìm hiểu về lãnh đạo đạo đức và văn hoá tổ chức ảnh hưởng thế nào đến sự cam kết của nhân viên trong ngành du lịch tại Thành phố Hồ Chí Minh. Hoặc nghiên cứu của Yuan, Vu, và Nguyen (2018) nhằm tìm hiểu mối quan hệ giữa lãnh đạo đạo đức, sự trao đổi lãnh đạo - nhân viên và hành vi lời nói của nhân viên trong lĩnh vực dịch vụ. Tương tự vậy, các nghiên cứu về sự hoài nghi đối với tổ chức của người lao động vẫn chưa được đào sâu hay khám phá. Nhìn chung, các nghiên cứu dạng này vẫn đang định hướng về khái niệm lòng tin đối với tổ chức với các tiền tố và hệ quả của nó, mà chưa chú trọng vào các yếu tố tiêu cực khác cũng không kém phần quan trọng. Điển hình như nghiên cứu của Luu (2012), được thực hiện với các 214 giám đốc và quản lý bán hàng, mục tiêu của nghiên cứu là tìm hiểu về mối quan hệ giữa các phong cách lãnh đạo, lòng tin đối với tổ chức và các yếu tố khác, và vấn đề về sự hoài nghi đối với tổ chức vẫn chưa được đề cập đến. Từ các thực tế trên có thể nhận thấy rằng các vấn đề nổi cộm gần đây của thế giới như các mặt tiêu cực của tổ chức, thì tại Việt Nam vẫn chưa nhận được một sự quan tâm tương xứng, trong khi các ảnh hưởng này ngày càng được chứng minh và thể hiện rõ nét trên thế giới. Điều này rất quan trọng vì nếu đây là một lổ hổng nghiên cứu lớn thì hiển nhiên các nghiên cứu ứng dụng hay giải pháp khắc phục và phòng ngừa là còn rất hạn chế.

Vì những lý do kể trên, nghiên cứu này được thực hiện với các mục tiêu chính được đề ra như sau: (1) kiểm định lại mối quan hệ giữa lãnh đạo tư lợi, sự hoài nghi đối với tổ chức và sự kiệt sức về tinh thần của người lao động trong các doanh nghiệp tại Thành phố Hồ Chí Minh; (2) đánh giá đồng thười so sánh các tác động này với nhau, và kiểm định vai trò của các biến điều tiết nếu có. Các kết quả nghiên cứu có được là cơ sở để đề xuất các hàm ý quản trị liên quan nhằm làm giảm các ảnh hưởng tiêu cực liên quan có thể có đến người lao động trong tổ chức.

\section{Cơ sở lý thuyết và giả thuyết nghiên cứu}

Thuyết duy trì nguồn lực (Conservation of Resources) là một lý thuyết về tâm lý học được đề xuất bởi tiến sĩ Stevan $\mathrm{E}$. Hobfoll năm 1989 và được ứng dụng nhiều trong các nghiên cứu về căng thẳng tâm lý (stress) nói chung, stress trong công việc nói riêng, hội chứng cạn kiệt sức lực vì công việc (burnout) hay cụ thể hơn là sự kiệt sức về tinh thần (emotional exhaustion). Thuyết này được đề xuất nhằm mục đích giải thích cho tình trạng căng thẳng tâm lý của con người nói chung, nguyên nhân chính được cho là việc người nào đó cùng lúc duy trì nguồn lực hiện có của mình và đồng thời cũng theo đuổi các nguồn lực mới. Thông qua nghiên cứu của mình, Hobfoll (1989) đã khẳng định rằng sự căng thẳng tâm lý xảy ra trong ba trường hợp: thứ nhất khi có một mối đe dọa nào đó có thể làm mất mát nguồn lực, thứ hai khi thực sự xảy ra việc mất mát nguồn lực, và cuối cùng là khi tiêu tốn cho nguồn lực nào đó nhưng kết quả sau đó lại không đạt được hoặc không có nguồn lực khác bù vào. Theo Halbesleben, Neveu, Paustian-Underdahl, và Westman (2014) các nguồn lực mà Hobfoll đề cập bên trên có thể được định nghĩa cụ thể là: giá trị của một ai đó, một đối tượng cụ thể nào đó, và địa vị xã hội hoặc các điều kiện của người đó.

Dựa trên nền tảng của thuyết duy trì nguồn lực, nghiên cứu tổng thể của Lee và Ashforth (1996) đã được thực hiện nhằm kiểm chứng mối quan hệ tương quan giữa nhu cầu và nguồn lực, giữa hành vi và thái độ, và ba thành phần của sự cạn kiệt sức lực trong công việc. Nguyên tắc đầu tiên của thuyết, được gọi là "Nguyên tắc ưu tiên với mất mát nguồn lực" (Primacy of Resource 
Loss) đã được phát biểu trong nghiên cứu này. Theo nguyên tắc này, khi nguồn lực của cá nhân nào đó bị mất đi, cá nhân có xu hướng nhạy cảm và cảm thấy bị tổn thương với sự mất mát này. Điều này cũng có nghĩa là dù có đạt được lợi ích từ chính việc mất mát này đi chăng nữa thì cá nhân đó vẫn cảm thấy bị tổn hại. Cũng dựa vào lý thuyết mà Hobfoll (1989) đề xuất, nghiên cứu về sau của Halbesleben và cộng sự (2014) cũng cho kết quả phù hợp với phù hợp nghiên cứu của Lee và Ashforth (1996). Ngoài ra, nghiên cứu này còn đề xuất nguyên tắc thứ hai của thuyết, được gọi là "Nguyên tắc đầu tư nguồn lực" (Resource Investment). Theo nguyên tắc này, con người sẽ có khuynh hướng đầu tư vào nguồn lực để bảo vệ chống lại sự mất mát với các nhiều động cơ, có thể kể đến như: ngăn ngừa tổn thất trong tương lai, phục hồi từ sự mất mát, hoặc để đạt được nguồn lực nào đó.

Lý thuyết ban đầu mà Hobfoll (1989) đề xuất nhằm cố gắng giải thích về hành vi cá nhân. Tuy nhiên các nghiên cứu về sau (Cropanzano et al., 2003; Wright \& Cropanzano, 1998) dựa trên nền tảng của thuyết này chỉ nhằm giải thích về stress của cá nhân trong đời sống hàng ngày và công việc, thậm chí xa hơn là về thái độ của nhân viên trong công việc. Nhìn chung, các nhà nghiên cứu thường sử dụng kết hợp với các thuyết khác khi nghiên cứu về stress tâm lý của con người nói chung, hoặc người lao động nói riêng trong công việc và đời sống, có thể kể đến như sau. Nghiên cứu của Cropanzano và cộng sự (2003) đã sử dụng kết hợp thuyết này với Thuyết trao đổi xã hội (Social Exchange Theory). Nghiên cứu của Becker, Halbesleben, và Dan O'Hair (2005) sử dụng kết hợp thuyết này với Thuyết trao đổi lãnh đạo - thành viên (Leader - Member Exchange Theory). Tuy nhiên, cũng có một số nhà nghiên cứu (Halbesleben et al., 2014; Lee \& Ashforth, 1996; Wright \& Cropanzano, 1998) đề xuất rằng khi nghiên cứu về sự cạn kiệt sức lực trong công việc thì nên đặt thuyết này ở vị trí trọng tâm.

\subsection{Hội chúng cạn kiệt sức lục}

Hội chứng kiệt sức lực vì công việc (burnout) là một khái niệm nghiên cứu liên quan đến stress công việc (work - related stress), và cũng là một khái niệm nghiên cứu dựa trên nền tảng của thuyết duy trì nguồn lực như đã đề cập ở phần trên. Có thể hiểu ngắn gọn đó là việc chịu đựng stress trong thời gian dài dẫn đến hiện tượng này (Cordes \& Dougherty, 1993; Maslach, Jackson, Leiter, Schaufeli, \& Schwab, 1986). Theo định nghĩa của Maslach và Jackson (1981) thì sự cạn kiệt sức lực là một hội chứng tâm lý bao gồm: (a) sự kiệt sức về tinh thần (emotional exhaustion); (b) mất ý chí (depersonalization) và (c) đánh giá thấp thành tựu cá nhân (reduced personal accomplishment). Gần đây nhất, nhằm góp phần đưa ra những định hướng về sức khỏe tâm lý tại nơi làm việc và cảnh báo về sự gia tăng của hội chứng này, Tổ Chức Y Tế Thế Giới - World Health Organization WHO (2019) đã xem đây là không chỉ là một hội chứng về tâm lý mà còn là liên quan đến cả thế chất và tinh thần của người lao động, và được gọi là Hội chứng cạn kiệt sức lực (burnout syndrome).

\subsection{Sụ kiệt sức về tinh thần}

Các nghiên cứu trước đây cũng đã chỉ ra hai điều rằng: (1) sự kiệt sức về tinh thần của nhân viên thể hiện mạnh mẽ, nhất quán và rõ ràng hơn dựa trên kết quả nghiên cứu thực tiễn (Cropanzano et al., 2003; Maslach et al., 2001); và (2) nó quan trọng hơn khi so với hai thành phần còn lại của hội chứng cạn kiệt sức lực (Bakker \& Costa, 2014). Theo định nghĩa của Maslach và cộng sự (1986), sự kiệt sức về tinh thần diễn ra khi các nguồn lực về tinh thần bị rút kiệt và người lao động cảm thấy họ không thể ổn định tâm lý ở một mức độ nào đó. Demerouti, Bakker, Nachreiner, và Schaufeli (2001) nhận thấy sự kiệt sức về tinh thần cũng bao gồm các cảm giác từ bỏ và mắc kẹt; theo đó cá nhân phát triển một thái độ tiêu cực đối với bản thân, công việc và cuộc sống của họ; kết quả là người chịu cảm giác này thì sau sáu đến tám tuần căng thẳng dữ dội dẫn 
đến sự mệt mỏi về thể chất và mất khả năng điều khiển các tình huống, thể hiện tất cả các triệu chứng của hội chứng cạn kiệt sức lực.

\subsection{Lãnh đạo tur lọi}

Các nghiên cứu gần đây (Chiaburu et al., 2013; LeBreton et al., 2018; Thundiyil, Chiaburu, Oh, Banks, \& Peng, 2015) cho thấy khi xét đến các tiền tố gây nên các tác động tiêu cực với tổ chức thì lãnh đạo là một nhân tố không thể bỏ qua. Theo Gregoire và Arendt (2004) có nhiều cách tiếp cận về lãnh đạo, và cách tiếp cận về phẩm chất/ đặc tính của nhà lãnh đạo cần phải được lưu tâm và nghiên cứu nhiều hơn (Dionne et al., 2014; Yammarino, Dionne, Chun, \& Dansereau, 2005). Theo Furtner, Maran, và Rauthmann (2017); Paulhus và Williams (2002), khi nói về các mặt tối của nhân cách con người hầu hết các nhà nghiên cứu thường sử dụng khái niệm ba mặt tối của nhân cách (Dark Triad), cụ thể là: ái kỷ (Narcissism), tư lợi (Machiavellianism) và thái nhân cách (Psychopathy). Christie và Geis (1970) đã đầu tiên sử dụng thuật ngữ nhân cách tư lợi (Machiavellianism) nhằm phản ánh nhân cách của một nhân vật có thật trong lịch sử tên là Niccolò Machiavelli. LeBreton và cộng sự (2018) cho rằng mặc dù có các định nghĩa khác nhau nhân cách tư lợi, nhưng đều cùng công nhận rằng đặc tính cốt lõi đó là xu hướng thao túng người khác. Cụ thể là thể hiện các đặc tính như: (1) xu hướng thao túng người khác, (2) thiếu sự đồng cảm, (3) mức độ bị ảnh hưởng bởi người khác thấp hơn, (4) tập trung vào việc theo đuổi mục tiêu của riêng của mình và (5) một cái nhìn bất thường về đạo đức.

Các nghiên cứu trước đây về lãnh đạo tư lợi cho thấy, các nhà lãnh đạo thuộc nhóm nhân cách này có tác động lên tổ chức và nhân viên của họ ở nhiều khía cạnh, trong đó có sự kiệt sức về tinh thần (LeBreton et al., 2018). Vấn đề về lãnh đạo tư lợi được làm rõ hơn qua nghiên cứu của Volmer và cộng sự (2016), nghiên cứu này chỉ ra rằng lãnh đạo tư lợi tác động cùng chiều lên sự kiệt sức về tinh thần của nhân viên, trong khi lãnh đạo ái kỷ và thái nhân cách lại tác động đến các mặt khác của tổ chức. Trên cơ sở đó, tác giả đề xuất giả thuyết nghiên cứu như sau:

\section{H1: Lãnh đạo tu lơi có tác động trực tiếp cùng chiều đến sự kiệt sức về tinh thần của nhân viên}

\subsection{Sự hoài nghi đối với tổ chức}

Tương tự như sự kiệt sức về tinh thần, sự hoài nghi của người lao động đối với tổ chức (organizational cynicism) cũng là một yếu tố tiêu cực của tổ chức, nó thể hiện thái độ tiêu cực của người lao động trong tổ chức, và được xem như là tương phản với lòng tin với tổ chức (trust in organization). Sự hoài nghi đối với tổ chức có thể có nhiều cách tiếp cận, có thể do sự hoài nghi từ trong chính nhân cách của người đó hoặc từ khía cạnh khác của bản thân người đó. Dean và cộng sự (1998) đã tổng hợp các nghiên cứu trước đó và đầu tiên đưa ra định nghĩa về sự hoài nghi đối với tồ chức. Theo đó, sự hoài nghi với tổ chức là một thái độ tiêu cực hướng về tổ chức người đó đang làm việc, bao gồm ba thành phần: (1) niềm tin của người nào đó rằng tổ chức của họ thiếu tính toàn vẹn; (2) tác động tiêu cực của người đó hướng về tổ chức; (3) xu hướng hành vi có thể làm ô danh hoặc các hành vi nguy hiểm đối với tổ chức (xu hướng này được xem là kết quả hoà trộn từ niềm tin và tác động trên).

Theo các nhà nghiên cứu khi các kỳ vọng của người lao động không được đáp ứng sẽ dẫn đến hệ quả tiêu cực, trong đó có sự hoài nghi đối với tổ chức. Các nghiên cứu tổng quan gần đây (Chiaburu et al., 2013; Thundiyil et al., 2015) đã đề xuất rằng sự hoài nghi đối với tổ chức sẽ làm gia tăng sự kiệt sức về tinh thần của người lao động. Kết quả nghiên cứu của Johnson và O'LearyKelly (2003) đã khẳng định sự hoài nghi đối với tổ chức ngoài các tác động tiêu cực đến thái độ của nhân viên, trong đó có sự kiệt sức về tinh thần. Gần đây, nghiên cứu của Naseer, Raja, và Syed (2017) cũng chỉ ra rằng sự hoài nghi đối với tổ chức tác động cùng lên trực tiếp lên sự kiệt sức về tinh thần của nhân viên thông qua biến điều tiết là yếu tố tâm lý. Trên cơ sở đó, giả thuyết nghiên cứu được đề xuất như sau: 
H2: Sự hoài nghi đối với tổ chức có tác động trục tiếp cùng chiều với sụ kiệt sức về tinh thần của nhân viên

\subsection{Vai trò trung gian của sụ hoài nghi đối với tổ chức}

Khi nghiên cứu các tác động của lãnh đạo tư lợi và sự hoài nghi đối với tổ chức lên sự kiệt sức về tinh thần Gkorezis, Petridou, và Krouklidou (2015) đã nhận thấy rằng ngoài các tác động trực tiếp như đã đề cập trên, sự hoài nghi đối với tổ chức còn đóng một vai trò trung gian bổ sung trong mối quan hệ giữa lãnh đạo tư lợi và sự kiệt sức về tinh thần. Điều này được khẳng định một lần nữa qua nghiên cứu của Stradovnik và Stare (2018). Kết quả của nghiên cứu này cho thấy, hai tác động trực tiếp cùng chiều của lãnh đạo tư lợi và sự hoài nghi đối với tổ chức lên sự kiệt sức về tinh thần là có xuất hiện nhưng chưa đủ mạnh bằng tác động gián tiếp. Vì vậy trong nghiên cứu này, giả thuyết nghiên cứu được đề ra như sau:

H3: Sụ hoài nghi đối với tổ chức giữ vai trò trung gian bổ sung trong mối quan hệ giũa lãnh đạo tu lợi và sụ kiệt sức về tinh thần của nhân viên

\section{Phương pháp nghiên cứu}

Phương pháp nghiên cứu được sử dụng là kết hợp giữa phương pháp nghiên cứu định tính và định lượng. Kế thừa từ các nghiên cứu trước đây, các thang đo gốc được sử dụng lại để làm cơ sở, và từ đó thiết kế bảng câu hỏi và thu thập thông tin sơ cấp. Sau đó, tác giả phân tích dữ liệu bằng cách sử dụng phần mềm SPSS 20.0 và AMOS 24.0.

\section{1. Đo lường}

Khái niệm lãnh đạo tư lợi sẽ được đo lường thông qua cảm nhận của nhân viên cấp dưới trực thuộc người lãnh đạo đó, và được đo lường bằng Short Dark Triad (SD3) được xây dựng bởi Jones và Paulhus (2014) bao gồm 09 phát biểu. Khái niệm sự kiệt sức về tinh thần của nhân viên được đo lường bằng thang Maslach Burnout Inventory $(\mathrm{MBI})$, được xây dựng bởi Maslach và cộng sự (2001) bao gồm 09 phát biểu. Khái niệm sự hoài nghi đối với tổ chức của nhân viên được đo lường bằng thang đo của Brandes, Dharwadkar, và Dean (1999) với 11 biến quan sát. Các thang đo lường gốc của các nghiên cứu trước đây được thể hiện chi tiết trong phần Phụ Lục.

Các thang đo này trước hết cần phải điều chỉnh bằng thảo luận cặp đôi để có thể phù hợp hơn với bối cảnh tại Việt Nam. Thảo luận cặp đôi được thực hiện từ tháng 08/2020 đến tháng 09/2020. Số lượng tham gia thảo luận cặp đôi là 10 người. Kết quả thảo luận cặp đôi cho thấy không có sự loại trừ hay bổ sung thêm các phát biểu mới so với các thang đo gốc, ngoại trừ việc hiệu chỉnh về mặt dịch thuật và bổ sung các từ ngữ nhằm làm rõ ý nghĩa của các phát biểu. Tất các phát biểu được đo lường bởi thang đo Likert -7 , từ rất không đồng ý đến rất đồng ý. Các thang đo lường được hiệu chỉnh cho phù hợp để được sử dụng trong nghiên cứu này, chủ yếu là chỉnh sửa về mặt từ ngữ và dịch thuật.

\subsection{Thu thập dĩu liệu}

Hiện tại, Thành phố Hồ Chí Minh đang là một ba thành phố lớn của Việt Nam, đồng thời cũng là một trong những trung tâm văn hoá - kinh tế lớn của cả nước. Trước những đòi hỏi ngày càng cao của môi trường công việc năng động này, người lao động tại đây sẽ phát sinh ngày càng nhiều những vấn đề về sức khỏe thể chất lẫn tinh thần. Vì lẽ đó Thành phố Hồ Chí Minh được xem như nơi có tiềm năng để nghiên cứu về những vấn đề khá mới này. Đối tượng khảo sát được chọn là người lao động đang làm việc tại Thành phố Hồ Chí Minh, họ đến từ nhiều ngành nghề và lĩnh vực hoạt động và phù hợp với sự đa dạng về ngành nghề. Tổng số phiếu khảo sát trực tiếp được phát là 362 phiếu, số phiếu thu về là 344 phiếu, đạt tỷ lệ trả lời là $95.0 \%$. Trong đó, số phiếu không đạt yêu cầu là 08 phiếu, do trả lời là hiện đang không làm việc tại Thành phố Hồ Chí Minh. 
Vì vậy, số phiếu hợp lệ là 336 phiếu, tỷ lệ so với tổng số phiếu phát hành là $92.8 \%$. Các khảo sát này được thực hiện từ ngày $01 / 10 / 2020$ đến 31/10/2020.

\section{Kết quả và thảo luận}

\subsection{Kết quả nghiên cúu}

Kết quả phân tích số liệu sẽ được trình bày theo bốn phần: (1) đặc điểm mẫu lựa chọn được phân tích, (2) kiểm định thang đo, (3) ước lượng các mối quan hệ cấu trúc trong mô hình nghiên cứu và kết quả này được sử dụng để kiểm định các giả thuyết đã đề ra, và (4) phân tích vai trò của các biến điều tiết.

\subsection{1. Đặc điểm mẫu thống kê}

Mẫu khảo sát trong trong nghiên cứu này có các đặc điểm như sau. Xét về giới tính, $52.4 \%$ là nam và $47.6 \%$ là nữ. Về độ tuổi tập trung từ 23 - 32 tuổi, chiếm $75.3 \%$. Về vị trí công tác chủ yếu nhân viên chiếm $68.8 \%$. Ngoài các yếu tố về nhân khẩu học, nghiên cứu này cũng thực hiện một khảo sát sơ bộ về nhận thức và biểu hiện của sự kiệt sức về tinh thần. Kết quả từ số liệu cho thấy, nếu tính theo tần suất xuất hiện theo tuần, thì nhóm những người chưa bao giờ nghe qua sự kiệt sức về tinh thần và hội chứng cạn kiệt sức lực lại thể hiện các triệu chứng của sự kiệt sức này nhiều hơn nhóm còn lại. Điều này cũng cho thấy, cho dù sự kiệt sức về tinh thần và hội chứng cạn kiệt sức lực là một khái niệm mới và chưa được chú ý nhiều nhưng các biểu hiện của nó lên người lao động là một vấn đề cần phải lưu tâm.

\subsubsection{Kiểm định thang đo}

Một cách tổng quát, các thang đo được sử dụng có hệ số Cronbach's alpha tổng dao động trong khoảng từ 0.828 đến 0.942 ; đều lớn hơn 0.6 và đảm bảo độ tin cậy cần thiết. Về hệ số tương quan biến tổng dao động trong khoảng từ 0.635 đến 0.883 ; đều lớn hơn 0.3 và đạt chuẩn cần thiết. Khi loại một trong các biến này thì hệ số Cronbach's alpha tổng tương ứng của thang đó đó đều thấp hơn ban đầu. Do vậy, các biến quan sát trong các thang đo này được giữ nguyên do chúng đảm bảo độ tin cậy.

Tiếp theo, trong phân tích nhân tố khám khá (EFA - Exploratory Factor Analysis), với phép trích PCA (Principal Component Analysis) và phép xoay vuông góc Varimax, kết quả thu được như sau. Đầu tiên, phân tích EFA riêng cho hai khái niệm đơn hướng là lãnh đạo tư lợi ( 09 biến quan sát) và sự kiệt sức về tinh thần (09 biến quan sát). Về trọng số nhân tố, các biến đều có trọng số nhân tố $>0.4$. Về tổng phương sai trích TVE là $66.491 \%>50 \%$. Về số lượng nhân tố trích, hệ số Eigenvalue là $3.102>1$. Về kiểm định $\mathrm{KMO}$ và Bartlett, hệ số $\mathrm{KMO}$ là $0.94>0.5$ và Sig. (mức ý nghĩa của kiểm định Bartlett) $<0.05$. Sau đó, phân tích EFA được thực hiện riêng cho khái niệm đa hướng là sự hoài nghi đối với tổ chức với 11 biến quan sát, bao gồm ba thành phần là cảm xúc hoài nghi, nhận thức hoài nghi và hành động hoài nghi. Về trọng số nhân tố, các biến đều có trọng số nhân tố $>0.4$. Về tổng phương sai trích TVE là $76.615 \%>50 \%$. Về số lượng nhân tố trích, hệ số Eigenvalue là $1.273>1$. Về kiểm định $\mathrm{KMO}$ và Bartlett, hệ số $\mathrm{KMO}$ là $0.863>0.5$ và Sig. (mức ý nghĩa của kiểm định Bartlett) $<0.05$. Từ đó, có thể kết luận rằng các kết quả trên phù hợp với các điều kiện của $\mathrm{EFA}$, và các biến quan quan sát đều được giữ lại để tiến hành cho các bước phân tích tiếp theo.

Cuối cùng, kết quả phân tích nhân tố khẳng định (CFA - Confirmatory Factor Analysis) cho thấy. Về các chỉ số phù hợp mô hình, kết quả CFA cho thấy Chi-square/df $(\mathrm{Cmin} / \mathrm{df})=1.984$; $\mathrm{p}=0.000 ; \mathrm{CFI}=0.951 ; \mathrm{TLI}=0.945, \mathrm{SRMR}=0.050 ; \mathrm{RMSEA}=0.054$ và $\mathrm{pClose}=0.117$. Như vậy, sự phù hợp với các dữ liệu thị trường của của các thành phần trong mô hình tới hạn này là thỏa yêu cầu. Kết quá CFA của mô hình cũng cho thấy, độ tin cậy thang đo và giá trị hội tụ cũng thỏa được các điều kiện yêu cầu, thể hiện qua Hình 1 như sau. 


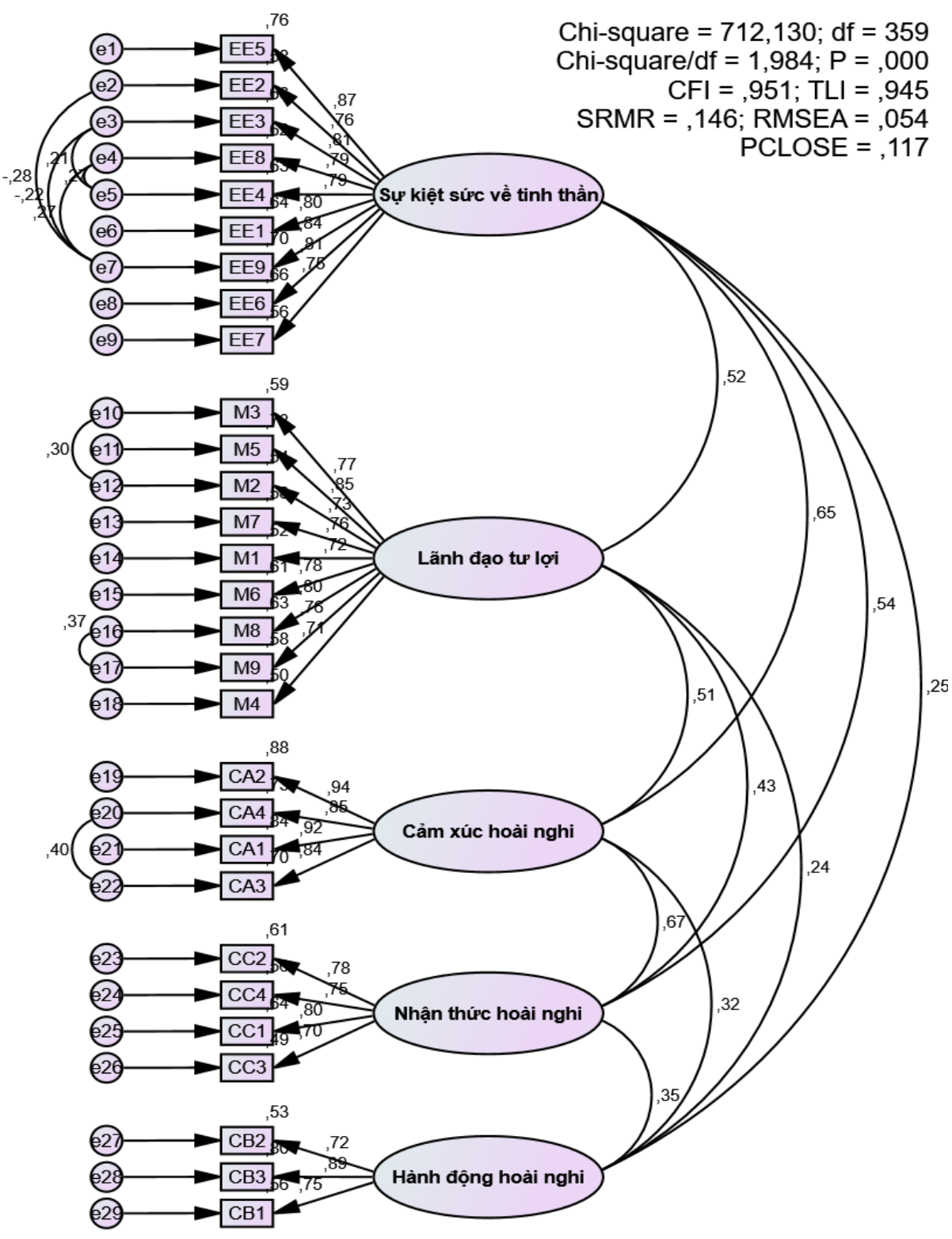

Hình 1. Kết quả CFA (chuẩn hóa)

Mô hình CFA tới hạn ở Hình 1 cũng đạt được giá trị phân biệt khi thỏa điều kiện MSV < $A V E$ và căn bậc hai $A V E>$ các giá trị tương quan của nó với các khái niệm khác. Ngoài ra, tất cả các mối tương quan đều có hệ số tương quan khác biệt so với 1 và có ý nghĩa thống kê với độ tin cậy $99 \%$. Kết quả được thể hiện qua Bảng 1 và Bảng 2 như sau.

\section{Bảng 1}

Kiểm định giá trị phân biệt giữa các thành phần trong mô hình tới hạn

\begin{tabular}{|c|c|c|c|c|c|c|c|c|c|}
\hline & $\mathbf{C R}$ & AVE & $\overline{\text { MSV }}$ & $\operatorname{MaxR}(H)$ & $\overline{\mathbf{E E}}$ & $\mathbf{M}$ & $\mathbf{C C}$ & CB & $\overline{\mathbf{C A}}$ \\
\hline $\mathbf{E E}$ & 0,942 & 0,645 & 0,42 & 0,945 & 0,803 & - & - & - & - \\
\hline $\mathbf{M}$ & 0,928 & 0,588 & 0,268 & 0,931 & $0,518 * * *$ & 0,767 & - & - & - \\
\hline $\mathbf{C C}$ & 0,936 & 0,787 & 0,447 & 0,946 & $0,648 * * *$ & $0,514 * * *$ & 0,887 & - & - \\
\hline CB & 0,844 & 0,576 & 0,447 & 0,848 & $0,535 * * *$ & $0,431 * * *$ & $0,668 * * *$ & 0,759 & - \\
\hline CA & 0,834 & 0,627 & 0,122 & 0,864 & $0,248 * * *$ & $0,236 * * *$ & $0,321 * * *$ & $0,350 * * *$ & 0,792 \\
\hline
\end{tabular}

Nguồn: Xử lý số liệu của tác giả 


\section{Bảng 2}

Tương quan giữa các thành phần trong mô hình tới hạn

\begin{tabular}{|c|c|c|c|c|}
\hline Mối quan hệ & Tưong quan & $\begin{array}{l}\text { Sai số chuẩn } \\
\text { (SE) }\end{array}$ & $\begin{array}{l}\text { Giá trị tới hạn } \\
\text { (C.R) }\end{array}$ & p-value \\
\hline $\begin{array}{l}\text { Sự kiệt sức về tinh thần } \\
\text { <--> Lãnh đạo tư lợi }\end{array}$ & 0,945 & 0,129 & 7,321 & $* * *$ \\
\hline $\begin{array}{l}\text { Sự kiệt sức về tinh thần } \\
\quad<->\text { Cảm xúc hoài nghi }\end{array}$ & 1,623 & 0,179 & 9,090 & $* * *$ \\
\hline $\begin{array}{l}\text { Sự kiệt sức về tinh thần } \\
\quad<-->\text { Nhận thức hoài nghi }\end{array}$ & 0,832 & 0,113 & 7,339 & $* * *$ \\
\hline $\begin{array}{l}\text { Sự kiệt sức về tinh thần } \\
\quad<-->\text { Hành động hoài nghi }\end{array}$ & 0,410 & 0,106 & 3,871 & $* * *$ \\
\hline $\begin{array}{l}\text { Lãnh đạo tư lợi } \\
\quad<-->\text { Cảm xúc hoài nghi }\end{array}$ & 1,250 & 0,169 & 7,385 & $* * *$ \\
\hline $\begin{array}{l}\text { Lãnh đạo tư lợi } \\
\quad<-->\text { Nhận thức hoài nghi }\end{array}$ & 0,651 & 0,108 & 6,053 & $* * *$ \\
\hline $\begin{array}{l}\text { Lãnh đạo tư lợi } \\
\quad<-->\text { Hành động hoài nghi }\end{array}$ & 0,380 & 0,104 & 3,640 & $* * *$ \\
\hline $\begin{array}{l}\text { Cảm xúc hoài nghi } \\
\quad<-->\text { Nhận thức hoài nghi }\end{array}$ & 1,384 & 0,160 & 8,646 & $* * *$ \\
\hline $\begin{array}{l}\text { Cảm xúc hoài nghi } \\
\quad<-->\text { Hành động hoài nghi }\end{array}$ & 0,708 & 0,146 & 4,861 & $* * *$ \\
\hline $\begin{array}{l}\text { Nhận thức hoài nghi } \\
\quad<-->\text { Hành động hoài nghi }\end{array}$ & 0,479 & 0,098 & 4,914 & $* * *$ \\
\hline
\end{tabular}

Nguồn: Xử lý số liệu của tác giả

\subsection{3. Ước lương các mối quan hệ cấu trúc}

\subsubsection{Kiểm định mô hình lý thuyết bằng SEM}

Hình 2 trình bày kết quả phân tích SEM để kiểm định giả thuyết cho mô hình nghiên cứu. Kết quả cho thấy mô hình đạt được sự phù hợp về dữ liệu với các chỉ số như sau: Chi-square/df $(\mathrm{Cmin} / \mathrm{df})=2.064 ; \mathrm{p}=0.000 ; \mathrm{CFI}=0.946 ; \mathrm{TLI}=0.945, \mathrm{SRMR}=0.052 ; \mathrm{RMSEA}=0.056$ và pClose $=0.034$. 


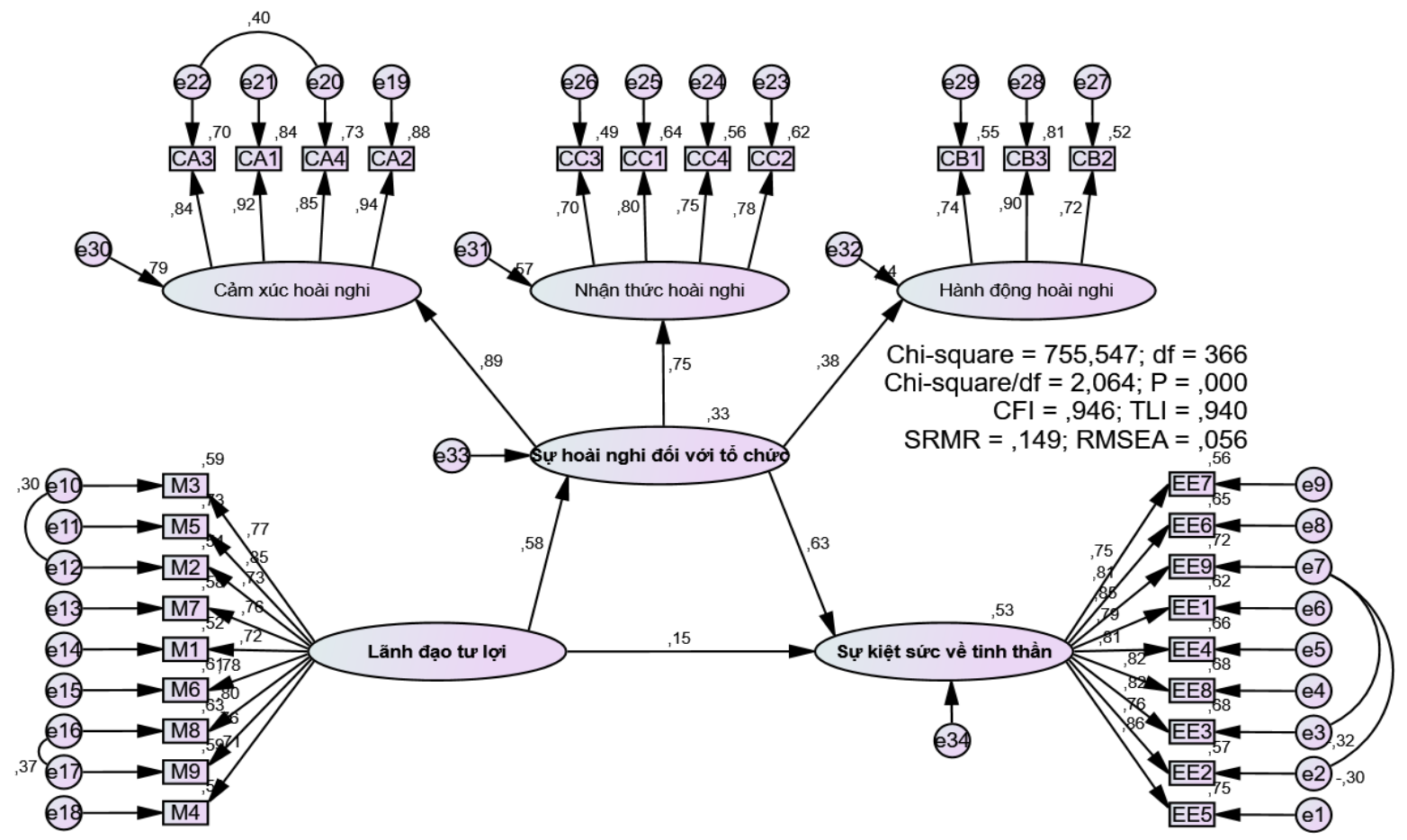

Hình 2. Kết quả phân tích SEM (chuẩn hoá)

\subsubsection{Kiểm định các giả thuyết của mô hình}

Bảng 3 trình bày kết quả ước lượng bằng Bootstrap số lượng mẫu lặp lại 2,000 lần. Kết quả cho thấy chỉ số tới hạn khi kiểm định độ lệch đều đạt yêu cầu, và tất cả các giả thuyết đề ra đều có ý nghĩa thống kê và với giá trị $\mathrm{p}<0.05$.

\section{Bảng 3}

Kết quả ước lượng bằng Bootstrap

\begin{tabular}{|l|c|c|c|c|c|}
\hline \multicolumn{1}{|c|}{ Tương quan } & $\begin{array}{c}\text { Giả } \\
\text { thuyết }\end{array}$ & $\begin{array}{c}\text { Giá trị } \\
\text { ước lượng } \\
\text { (ML) }\end{array}$ & $\begin{array}{c}\text { Sai số } \\
\text { chuẩn } \\
\text { (SE) }\end{array}$ & $\begin{array}{c}\text { Giá trị } \\
\text { tới hạn } \\
\text { (C.R) }\end{array}$ & p-value \\
\hline $\begin{array}{l}\text { Lãnh đạo tư lợi } \\
\text {---> Sự kiệt sức về tinh thần }\end{array}$ & $\mathrm{H} 1$ & 0,157 & 0,064 & 2,442 & 0,015 \\
\hline $\begin{array}{l}\text { Sự hoài nghi đối với tồ chức } \\
\text {---> Sự kiệt sức về tinh thần }\end{array}$ & $\mathrm{H} 2$ & 1,882 & 0,375 & 5,014 & $* * *$ \\
\hline $\begin{array}{l}\text { Lãnh đạo tư lợi } \\
--->\text { Sự hoài nghi đối với tổ chức }\end{array}$ & $\mathrm{H} 3$ & 0,198 & 0,039 & 5,007 & $* * *$ \\
\hline
\end{tabular}

Nguồn: Xử lý số liệu của tác giả

Về tác động trực tiếp, lãnh đạo tư lợi và sự hoài nghi đối với tổ chức đều tác động trực tiếp lên sự kiệt sức về tinh thần, và tác động của sự hoài nghi là mạnh hơn. Thêm nữa, lãnh đạo tư lợi cũng tác động trực tiếp lên sự hoài nghi đối với tổ chức, và tác động này là mạnh hơn so với lên sự kiệt sức về tinh thần. Về tác động gián tiếp, chỉ có lãnh đạo tư lợi là có tác động gián tiếp đến sự kiệt sức về tinh thần, thông qua vai trò trung gian của sự hoài nghi đối với tổ chức. Như vậy, ngoài tác động trực tiếp thì lãnh đạo tư lợi còn có tác động gián tiếp lên sự kiệt sức về tinh thần. Từ đó có thể kết luận rằng sự hoài nghi đối với tổ chức đóng vai trò là biến trung gian, cụ thể giữ 
vai trò là biến trung gian bổ sung (Complementary Mediation) trong mô hình nghiên cứu. Điều này có nghĩa là tác động của lãnh đạo tư lợi chỉ thể hiện trực tiếp một phần lên sự kiệt sức về tinh thần, phần còn lại sẽ thể hiện thông qua vai trò trung gian của sự hoài nghi, và bản thân sự hoài nghi cũng có tác động trực tiếp đến sự kiệt sức về tinh thần.

\subsubsection{Phân tích biến điều tiết}

\subsubsection{Phân tích quan hệ hồ tương}

Phân tích quan hệ hỗ tương giữa biến độc lập là cảm nhận về lãnh đạo tư lợi $(\mathrm{M})$ và các biến định lượng như: kinh nghiệm làm việc, thâm niên tại tổ chức và tuổi. Kết quả cho thấy ba biến định lượng như kinh nghiệm làm việc, thâm niên tại tổ chức và tuổi không có quan hệ hỗ tương với biến độc lập, nghĩa là 03 biến trên không đóng vai trò là biến điều tiết thuần tuý hay hỗn hợp.

\subsubsection{Phân tích biến điều tiết theo nhóm}

Phương pháp phân tích đa nhóm được sử dụng để kiểm định vai trò điều tiết cho hai biến định tính là giới tính, chức vụ. Về biến chức vụ hiện tại ở tổ chức, những người khảo sát được phân thành hai nhóm là nhân viên và quản lý. Kết quả cho thấy biến chức vụ hiện tại ở tổ chức không đóng vai trò là biến điều tiết theo nhóm. Về biến giới tính, những người khảo sát được phân thành hai nhóm là nam và nữ. Kết quả cho thấy nhóm nữ cho thấy tương tự như kết quả của nghiêm cứu chính thức. Tuy nhiên kết quả phân tích của nhóm giới tính nam lại có sự khác biệt. Cụ thể là với nhóm giới tính nam thì lãnh đạo tư lợi chỉ có tác động gián tiếp thông qua vai trò trung gian của sự hoài nghi đối với tổ chức, cho nên sự hoài nghi cũng thay đổi vai trò trung gian của mình từ trung gian bổ sung (Complementary mediation) sang trung gian chỉ có tác động gián tiếp (Indirect-only mediation). Điều này cho thấy giữa hai nhóm nam và nữ thì cảm nhận về lãnh đạo tư lợi có ảnh hưởng khác nhau. Kết quả được thể hiện qua Bảng 4 như sau.

\section{Bảng 4}

Kết quả phân tích đa nhóm về giới tính

\begin{tabular}{|c|c|c|c|c|c|}
\hline Tương quan & $\begin{array}{c}\text { Giả } \\
\text { thuyết }\end{array}$ & $\begin{array}{c}\text { Giá trị } \\
\text { ước lượng } \\
(\mathrm{ML})\end{array}$ & $\begin{array}{c}\text { Sai số } \\
\text { chuẩn } \\
\text { (SE) }\end{array}$ & $\begin{array}{c}\text { Giá trị } \\
\text { tới hạn } \\
\text { (C.R) }\end{array}$ & p-value \\
\hline \multicolumn{6}{|c|}{ Nhóm giới tính nam (tỷ lẹ 176/336) } \\
\hline $\begin{array}{l}\text { Lãnh đạo tư lợi } \\
\quad \text {---> Sự kiệt sức về tinh thần }\end{array}$ & $\mathrm{H} 1$ & 0,130 & 0,099 & 1,311 & 0,190 \\
\hline $\begin{array}{l}\text { Sự hoài nghi đối với tổ chức } \\
--->\text { Sự kiệt sức về tinh thần }\end{array}$ & $\mathrm{H} 2$ & 2,451 & 0,707 & 3,465 & $* * *$ \\
\hline $\begin{array}{l}\text { Lãnh đạo tư lợi } \\
\qquad-->\text { Sự hoài nghi đối với tổ chức }\end{array}$ & $\mathrm{H} 3$ & 0,218 & 0,056 & 3,877 & $* * *$ \\
\hline \multicolumn{6}{|c|}{ Nhóm giới tính nữ (tỷ lệ 160/336) } \\
\hline $\begin{array}{l}\text { Lãnh đạo tư lợi } \\
\quad \text {---> Sự kiệt sức về tinh thần }\end{array}$ & $\mathrm{H} 1$ & 0,177 & 0,082 & 2,151 & 0,031 \\
\hline $\begin{array}{l}\text { Sự hoài nghi đối với tổ chức } \\
--->\text { Sự kiệt sức về tinh thần }\end{array}$ & $\mathrm{H} 2$ & 1,315 & 0,359 & 3,665 & $* * *$ \\
\hline $\begin{array}{l}\text { Lãnh đạo tư lợi } \\
\qquad-->\text { Sự hoài nghi đối với tổ chức }\end{array}$ & $\mathrm{H} 3$ & 0,218 & 0,056 & 3,877 & $* * *$ \\
\hline
\end{tabular}

Nguồn: Xử lý số liệu của tác giả 


\subsection{Thảo luận các kết quả nghiên cứu}

Với mục tiêu của nghiên cứu đã đề ra, kết quả thu được cho thấy mối quan hệ giữa lãnh đạo tư lợi, sự hoài nghi đối với tổ chức, sự kiệt sức về tinh thần như sau.

Đầu tiên, hai giả thuyết được chấp nhận rằng lãnh đạo tư lợi và sự hoài nghi đối với tổ chức có tác động trực tiếp cùng chiều đến sự kiệt sức về tinh thần của người lao động. Kết quả này phù hợp với thuyết duy trì nguồn lực nói chung và các nghiên cứu đã thực hiện trước đây tại các bối cảnh khác về lãnh đạo tư lợi (LeBreton et al., 2018; Volmer et al., 2016) hay về sự hoài nghi đối với tổ chức (Johnson \& O'Leary- Kelly, 2003; Naseer et al., 2017). Trong trường hợp nếu lấy sự kiệt sức về tinh thần là trọng tâm, khi xét về mức độ thì tác động trực tiếp của sự hoài nghi đối với tổ chức $(\beta=0.629)$ là mạnh hơn với lãnh đạo tư lợi $(\beta=0.153)$. Trường hợp lấy lãnh đạo tư lợi là trọng tâm thì tác động trực tiếp của nó lên sự hoài nghi với tổ chức $(\beta=0.578)$ là mạnh hơn với lên sự kiệt sức về tinh thần $(\beta=0.153)$.

Tiếp theo, giả thuyết được chấp nhận rằng sự hoài nghi đối với tổ chức đóng vai trò trung gian bổ sung trong mối quan hệ giữa lãnh đạo tư lợi và sự kiệt sức về tinh thần. Kết quả này cũng phù hợp với kết quả các nghiên cứu trước đây (Gkorezis et al., 2015; Stradovnik \& Stare, 2018). Vai trò trung gian của sự hoài nghi đối với tổ chức là làm tăng cường tác động này lên ( $\beta$ tổng hợp $=0.516)$. Như vậy ảnh hưởng lãnh đạo tư lợi không thể hiện trực tiếp nhiều lên sự kiệt sức về tinh thần của nhân viên cấp dưới, mà thông qua vai trò trung gian của sự hoài nghi đối với tổ chức.

Cuối cùng, kết quả kiểm định biến điều tiết cho thấy có sự khác biệt giữa hai nhóm nam và nữ, cụ thể là sự kiệt sức về tinh thần nhóm nữ vừa chịu đồng thời tác động trực tiếp và gián tiếp của lãnh đạo tư lợi, còn nhóm nam thì chỉ chịu ảnh hưởng gián tiếp, và vai trò trung gian của sự hoài nghi đối với tổ chức vì thế cũng thay đổi theo. Kết quả này có sự khác biệt với các nghiên cứu trước đây, cụ thể là nghiên cứu của Gkorezis và cộng sự (2015) hay Stradovnik và Stare (2018) không tìm thấy sự khác biệt giữa các nhóm giới tính hay nhân khẩu, cũng như vai trò của các biến điều tiết khác. Điều này có thể giải thích là do sự khác biệt về văn hóa và các yếu tố về nhân khẩu học.

Một cách tổng quan, khi xét bối cảnh của nghiên cứu này, mối quan hệ giữa các khái niệm trong mô hình là có hiện hữu, và các kết quả nghiên cứu cũng phù hợp với các nghiên cứu trước được thực hiện tại những bối cảnh khác. Điều này cũng cho thấy các vấn đề sức khỏe tinh thần người lao động cũng như các mặt tiêu cực của tổ chức là một vấn đề cần phải lưu tâm trong tương lai.

\section{Kết luậnvà hàm ý quản trị}

\subsection{Kết luận}

Dựa vào các kết quả của nghiên cứu này và sự phù hợp với các nghiên cứu trước đây, có thể nhận thấy một số điều như sau. Đầu tiên, để phù hợp bối cảnh Việt Nam cụ thể là Thành phố Hồ Chí Minh, các thang đo gốc kế thừa từ các nghiên cứu trước đây được điều chỉnh về mặt từ ngữ và cũng đạt độ tin cậy cao. Tiếp theo, kết quả của nghiên cứu cũng phù hợp và tương tự với kết quả của các nghiên cứu trước đây đã thực hiện trên thế giới. Điều này chứng tỏ rằng không chỉ ở những bối cảnh khác mà tại Việt Nam, các mối quan hệ giữa các khái niệm này là có hiện hữu trong tổ chức. Cuối cùng, một cách tổng quan thì sự hoài nghi đối với tổ chức giữ vai trò trung gian bổ sung (partial mediation) trong mối quan hệ giữa lãnh đạo tự lợi và sự kiệt sức về tinh thần của người lao động, tuy nhiên có sự thay đổi về vai trò trung gian này giữa các nhóm giới tính.

\subsection{Hàm ý quản trị và các đề xuất}

Trên cơ sở có được từ kết quả của nghiên cứu, tác giả đề xuất một số hàm ý quản trị như sau. 
Thứ nhất, sự hoài nghi đối với tổ chức sẽ là nguyên nhân gây ra sự kiệt sức về tinh thần nhiều hơn so với lãnh đạo tư lợi. Khi xuất hiện sự kiệt sức về tinh thần của người lao động thì đầu tiên vấn đề cần được là sự hoài nghi đối với tổ chức vì thiếu hụt niềm tin là nguyên nhân chính gây nên sự kiệt sức về tinh thần. Tuy nhiên, các nguyên nhân về sự hoài nghi đối với tổ chức không chỉ đơn thuần là thuộc về lãnh đạo mà còn nhiều yếu tố khác. Vì vậy, việc làm giảm sự hoài nghi đối với tổ chức cần nhiều chiến lược phối hợp, và khi sự hoài nghi đối với tổ chức được giảm thiểu sẽ có tác động tích cực rất lớn với tổ chức.

Thứ hai, nhà lãnh đạo mang đặc tính tư lợi sẽ là một trong những nguyên nhân gây ra sự hoài nghi đối với tổ chức và sự kiệt sức về tinh thần của nhân viên cấp dưới. Thêm nữa, sự hoài nghi đối với tổ chức lại kéo theo các tác động tiêu cực khác không chỉ là sự kiệt sức về tinh thần. Do đó, khi tuyển dụng vào các vị trí lãnh đạo, tổ chức nên xem xét việc tuyển dụng các nhà lãnh đạo mang nhân cách tư lợi quá cao hoặc cao hơn bình thường. Tuy nhiên ở một khía cạnh khác các nhà lãnh đạo tư lợi cũng mang nhiều ưu điểm rất có lợi tổ chức. Vì vậy nên giải pháp có thể là phát triển kỹ năng lãnh đạo, thời tăng cường nhận thức và đào tạo các nhà lãnh đạo tư lợi, hay tăng cường giao tiếp của lãnh đạo với nhân viên bằng cách tạo dựng một môi trường giao tiếp thân thiện cởi mở nhằm ghi nhận ý kiến phản hồi tiêu cực của nhân viên.

Thứ ba, lãnh đạo tư lợi có thể làm gia tăng sự kiệt sức về tinh thần của nhân viên cấp dưới một cách trực tiếp và gián tiếp thông qua vai trò trung gian bổ sung của sự hoài nghi đối với tổ chức. Điều này cho thấy tác động tiêu cực của lãnh đạo tư lợi trong môi trường làm việc của tổ chức là âm thầm và khó thể hiện ngay. Ngoài ra, sự khác biệt của hai nhóm nam nữ trong nghiên cứu này cho thấy, nhóm nữ thể hiện một phần tác động của lãnh đạo tư lợi lên sự kiệt sức về tinh thần, còn nhóm nam không thể hiện trực tiếp. Vì vậy, trong nỗ lực hạn chế các hệ quả không mong muốn từ lãnh đạo tư lợi, tổ chức nên cân nhắc đến các chiến lược kết hợp một cách đồng bộ nhằm đạt được hiệu quả cao trong việc ứng dụng các giải pháp.

Các hàm ý quản trị đề xuất trên cũng góp phần cho thấy, các nhà quản trị cần có những cách tiếp cận và tư duy mới, từ đó đề những giải pháp mới nhằm hạn chế các mặt tiêu cực của tổ chức. Các giải pháp này được cân nhắc vì có những trường hợp sẽ các tác dụng tốt hơn nhiều so với các giải pháp truyền thống là gia tăng các hiệu ứng tích cực của tổ chức.

\subsection{Các giới hạn của nghiên cứu và kiến nghị}

Nghiên cứu này cũng tương tự như các nghiên cứu trước đây đã từng thực hiện, đó là có những mặt hạn chế của nó, có thể kể đến như sau. Thứ nhất đối tượng nghiên cứu chỉ là những người lao động tại Thành phố Hồ Chí Minh và không tập trung vào một ngành nghề cụ thể nào. Thứ hai là chọn mẫu thuận tiện và đối tượng khảo sát chủ yếu là những người lao động có trình độ cao, và cũng có thể chưa đại diện được cho những người lao động khác, như lao động phổ thông. Do đó, kết quả nghiên cứu này cũng cần được xem xét trong các khu vực hoặc thành phố khác, đối tượng có trình độ cũng như ngành nghề khác nhau, để đảm bảo tính khách quan và có thể khẳng định mạnh mẽ hơn. Mặt khác, nghiên cứu này chỉ xem xét mối quan hệ giữa 03 khái niệm, trong khi bản thân các khái niệm này đều có nhiều tiền đề và hệ quả kèm theo. Các nghiên cứu trước đây cũng chỉ ra các khái niệm nghiên cứu tiềm năng khác như: sự lạm dụng trong kiểm soát (abusive supervision) (Kiazad, Restubog, Zagenczyk, Kiewitz, \& Tang, 2010; Wisse \& Sleebos, 2016); các thái độ của người lao động như sự hài lòng công việc (Chiaburu et al., 2013; Volmer et al., 2016). Vì vậy để mở rộng phạm vi nghiên cứu, các nghiên cứu tiếp theo có thể cân nhắc và bổ sung các khái niệm khác vào mô hình nhằm có cái nhìn sâu sắc và đầy đủ hơn về những vấn đề còn khá mới này. 


\section{Tài liệu tham khảo}

Bakker, A., \& Costa, P. L. (2014). Chronic job burnout and daily functioning: A theoretical analysis. Burnout Research, 1(3), 112-119.

Baumeister, R. F., Bratslavsky, E., Finkenauer, C., \& Vohs, K. D. (2001). Bad is stronger than good. Review of General Psychology, 5(4), 323-370.

Becker, J. A., Halbesleben, J. R., \& Dan O'Hair, H. (2005). Defensive communication and burnout in the workplace: The mediating role of leader-member exchange. Communication Research Reports, 22(2), 143-150.

Brandes, P., Dharwadkar, R., \& Dean, J. W., Jr. (1999, May). Does organizational cynicism matter? Employee and supervisor perspectives on work outcomes. Eastern Academy of Management Proceedings, 2(1), 150-153.

Chiaburu, D. S., Peng, A. C., Oh, I.-S., Banks, G. C., \& Lomeli, L. C. (2013). Antecedents and consequences of employee organizational cynicism: A meta-analysis. Journal of Vocational Behavior, 83(2), 181-197.

Christie, R., \& Geis, F. L. (1970). Studies in Machiavellianism. Cambridge, MA: Academic Press.

Cordes, C. L., \& Dougherty, T. W. (1993). A review and an integration of research on job burnout. Academy of Management Review, 18(4), 621-656.

Cropanzano, R., Rupp, D. E., \& Byrne, Z. S. (2003). The relationship of emotional exhaustion to work attitudes, job performance, and organizational citizenship behaviors. Journal of Applied Psychology, 88(1), 160-169.

Dean, J. W., Jr., Brandes, P., \& Dharwadkar, R. (1998). Organizational cynicism. Academy of Management Review, 23(2), 341-352.

Demerouti, E., Bakker, A. B., Nachreiner, F., \& Schaufeli, W. B. (2001). The job demandsresources model of burnout. Journal of Applied Psychology, 86(3), 499-512.

Dionne, S. D., Gupta, A., Sotak, K. L., Shirreffs, K. A., Serban, A., Hao, C., . . Yammarino, F. J. (2014). A 25-year perspective on levels of analysis in leadership research. The Leadership Quarterly, 25(1), 6-35.

Furtner, M. R., Maran, T., \& Rauthmann, J. F. (2017). Dark leadership: The role of leaders' dark triad personality traits. In Leader development deconstructed (pp. 75-99). Cham: Springer.

Gkorezis, P., Petridou, E., \& Krouklidou, T. (2015). The detrimental effect of machiavellian leadership on employees' emotional exhaustion: Organizational cynicism as a mediator. Europe's Journal of Psychology, 11(4), 619-631.

Gregoire, M. B., \& Arendt, S. W. (2004). Leadership: Reflections over the past 100 years. Journal of the American Dietetic Association, 104(3), 395-403.

Grichnik, D., Smeja, A., \& Welpe, I. (2010). The importance of being emotional: How do emotions affect entrepreneurial opportunity evaluation and exploitation? Journal of Economic Behavior \& Organization, 76(1), 15-29.

Halbesleben, J. R. B., Neveu, J.-P., Paustian-Underdahl, S. C., \& Westman, M. (2014). Getting to the "COR" understanding the role of resources in conservation of resources theory. Journal of Management, 40(5), 1334-1364. 
Hobfoll, S. E. (1989). Conservation of resources. A new attempt at conceptualizing stress. American Psychologist, 44(3), 513-524.

Johnson, J. L., \& O'Leary- Kelly, A. M. (2003). The effects of psychological contract breach and organizational cynicism: Not all social exchange violations are created equal. Journal of Organizational Behavior: The International Journal of Industrial, Occupational and Organizational Psychology and Behavior, 24(5), 627-647.

Jones, D. N., \& Paulhus, D. L. (2014). Introducing the short Dark Triad (SD3): A brief measure of dark personality traits. Assessment, 21(1), 28-41.

Kiazad, K., Restubog, S. L. D., Zagenczyk, T. J., Kiewitz, C., \& Tang, R. L. (2010). In pursuit of power: The role of authoritarian leadership in the relationship between supervisors' Machiavellianism and subordinates' perceptions of abusive supervisory behavior. Journal of Research in Personality, 44(4), 512-519.

LeBreton, J. M., Shiverdecker, L. K., \& Grimaldi, E. M. (2018). The dark triad and workplace behavior. Annual Review of Organizational Psychology and Organizational Behavior, 5(1), 387-414.

Lee, R. T., \& Ashforth, B. E. (1996). A meta-analytic examination of the correlates of the three dimensions of job burnout. Journal of Applied Psychology, 81(2), 123-133.

Luu, T. T. (2012). The linkages among leadership, trust, and business ethics. Social Responsibility Journal, 8(1), 133-148.

Mai, K. N., \& Nguyen, N. V. Q. (2015). The effects of ethical leadership and organizational culture towards employees' sociability and commitment - A study of tourism sector in Ho Chi Minh city, Vietnam. Journal of Advanced Management Science, 3(4), 329-336.

Maslach, C., \& Jackson, S. E. (1981). The measurement of experienced burnout. Journal of Organizational Behavior, 2(2), 99-113.

Maslach, C., Jackson, S. E., Leiter, M. P., Schaufeli, W. B., \& Schwab, R. L. (1986). Maslach burnout inventory. Palo Alto, CA: Consulting Psychologists Press.

Maslach, C., Schaufeli, W. B., \& Leiter, M. P. (2001). Job burnout. Annual Review of Psychology, 52(1), 397-422.

Naseer, S., Raja, U., \& Syed, F. (2017). Combined effects of organizational cynicism and psychological capital on CWBs: A mod-med model. Paper presented at 77th Annual the Academy of Management Proceedings, Atlanta, Georgia, USA.

Nguyen, H. T. T., Kitaoka, K., Sukigara, M., \& Thai, A. L. (2018). Burnout study of clinical nurses in Vietnam: Development of job burnout model based on Leiter and Maslach's theory. Asian Nursing Research, 12(1), 42-49.

Paulhus, D. L., \& Williams, K. M. (2002). The dark triad of personality: Narcissism, Machiavellianism, and psychopathy. Journal of Research in Personality, 36(6), 556-563.

Spain, S. M., Harms, P., \& LeBreton, J. M. (2014). The dark side of personality at work. Journal of Organizational Behavior, 35(S1), S41-S60.

Stradovnik, K., \& Stare, J. (2018). Correlation between Machiavellian leadership and emotional exhaustion of employees: Case study: Slovenian municipalities. Leadership and Organization Development Journal, 39(8), 1037-1050. 
Thomas, N., \& Gupta, S. (2018). Organizational cynicism - What every manager needs to know. Development and Learning in Organizations, 32(2), 16-19.

Thundiyil, T. G., Chiaburu, D. S., Oh, I.-S., Banks, G. C., \& Peng, A. C. (2015). Cynical about change? A preliminary meta-analysis and future research agenda. The Journal of Applied Behavioral Science, 51(4), 429-450.

Volmer, J., Koch, I. K., \& Göritz, A. S. (2016). The bright and dark sides of leaders' dark triad traits: Effects on subordinates' career success and well-being. Personality and Individual Differences, 101, 413-418.

Wisse, B., \& Sleebos, E. (2016). When the dark ones gain power: Perceived position power strengthens the effect of supervisor Machiavellianism on abusive supervision in work teams. Personality and Individual Differences, 99, 122-126.

World Health Organization (WHO). (2019). Burn-out an "occupational phenomenon": International classification of diseases. Retrieved January 10, 2021, from https://www.who.int/news/item/28-05-2019-burn-out-an-occupational-phenomenoninternational-classification-of-diseases

Wright, T. A., \& Cropanzano, R. (1998). Emotional exhaustion as a predictor of job performance and voluntary turnover. Journal of Applied psychology, 83(3), 486-486.

Yammarino, F. J., Dionne, S. D., Chun, J. U., \& Dansereau, F. (2005). Leadership and levels of analysis: A state-of-the-science review. The Leadership Quarterly, 16(6), 879-919.

Yuan, L., Vu, C. M., \& Nguyen, N. T. T. (2018, January). Ethical leadership, leader-member exchange and voice behavior: Test of mediation and moderation processes. In Proceedings of the 2018 2nd International conference on management engineering, software engineering and service sciences, 33-42. 